\title{
2D and 3D Elemental Mapping of Biological Structures by Energy-Filtered TEM
}

\author{
R.D. Leapman, G. Zhang, L. Dorward, V.V. Speransky, and M.A. Aronova
}

Division of Bioengineering and Physical Science, ORS, National Institutes of Health, Bethesda, MD 20892

Over the past decade there have been significant advances in energy filtered transmission electron microscopy (EFTEM), which can now provide two-dimensional distributions of specific chemical elements with a higher sensitivity and higher spatial resolution than have been achievable previously $[1,2]$. By using tomographic methods it has recently become possible to extend elemental mapping into three dimensions, although this technique is quite new and is still under development [3, 4]. By using EFTEM, it is possible to detect physiologically relevant levels of phosphorus, sulfur and certain other elements in cellular regions that are a few nanometers in size $[5,6]$. For example, by imaging phosphorus it is feasible to map quantitatively the distribution of nucleic acid at a spatial resolution of about $8 \mathrm{~nm}$, as limited by beam damage, and with a detection limit (standard deviation) of approximately 20 base-pairs. Bound metal atoms, such as iron, can be imaged too with potential applications to the biology of metalloproteins or to the identification of specific metal labels attached to proteins.

For most applications of EFTEM elemental mapping, it is necessary to prepare freeze-substituted plastic-embedded sections that are unstained; frozen hydrated specimens are generally unable to withstand the required electron dose, except for studies of cells at very low spatial resolution. To obtain element-specific maps from sectioned cells, it is typically necessary to record two images, one at an energy loss just above the core edge and one at an energy loss just below the edge [1-6]. By carefully subtracting the pre-core edge background intensity from the post-core edge intensity, it is possible not only to determine the net core edge signal intensity but also to quantitate the number of atoms of a particular element in an image feature. Quantitative analysis of the core edge intensity requires collection of the zero-loss image, whose intensity corresponds to the total number of electrons transmitted through the specimen at each pixel; in addition, it is necessary to determine the partial inelastic scattering cross section for exciting a core electron into the energy window defined by the energy-selecting slit of the electron spectrometer [2].

By recording tilt series of energy-filtered images at energy losses above and below the core-edge of an element of interest, and by using established methods for tomographic reconstruction, it is possible to compute the elemental distribution in three dimensions. Automated elemental tomography is more complicated to perform than standard tomography and some special procedures must be employed to collect the data [7]. For example, it is often impracticable to correct for spatial drift and focus by using the pre-edge and post-edge images because gold fiducial particles deposited on the specimen surfaces for alignment might not be visible, or the particles might undergo contrast reversal over the tilt series due to plural scattering effects. To overcome this difficulty a zero-loss image is recorded for each tilt angle. It is necessary for the acquisition computer to control the spectrum energy offset (via the microscope accelerating voltage) and the width of the energyselecting slit. In addition, the acquisition system must control the tilt of the specimen goniometer, and the excitation of the image deflection coils and objective lens, as for standard electron tomography. 
An example of EFTEM tomography in Figure 1 shows the volume rendered phosphorus distribution in a sectioned nucleus of a Drosophila larval cell. Dual axis tilt series were acquired over a range of $\pm 66^{\circ}$ with increments of $3^{\circ}$, and images were acquired at $0 \mathrm{eV}, 120 \mathrm{eV}$ and $152 \mathrm{eV}$ for each tilt angle, with a total accumulated electron dose of approximately $10^{7}$ electrons per $\mathrm{nm}^{2}$. The $2 \mathrm{k} \mathrm{x} 2 \mathrm{k}$ pixel CCD camera was binned $x 4$ to provide 512 x 512 pixels and a pixel size of $2.7 \mathrm{~nm}$. The tilt series were aligned and the 3D volume reconstructed using the IMOD program from the University of Colorado [8]. The 3D volume was visualized using the Amira software (Mercury Computer Systems). The reconstructed phosphorus distribution provides information about the distribution of chromatin fibers in the nucleus, whereas dense phosphorus-containing structures in the top right of the reconstruction are attributed to ribosomal particles in a region of cytoplasm outside the nucleus. Such 3D elemental maps could reveal useful new information about the structure of the cell nucleus, which cannot be determined by conventional electron tomography of stained sections [9].

\section{References}

[1] L. Reimer, Energy-Filtering Transmission Electron Microscopy, Springer, Berlin, 1995.

[2] F. Hofer and P. Warbichler, Transmission Electron Energy Loss Spectrometry in Materials

Science and the EELS Atlas, 2nd ed., C. Ahn, ed., Wiley-VCH, Berlin, Chapter 6, 2004, p. 159.

[3] P.A. Midgley and M. Weyland, Ultramicroscopy 96 (2003) 413.

[4] R.D. Leapman et al., Ultramicroscopy 100 (2004) 115.

[5] F.P. Ottensmeyer, J. Ultrastruct. Res. 88 (1984) 121.

[6] D.P. Bazett-Jones et al., Science 264 (1994) 1134.

[7] M.A. Aronova et al., Biophys. J. abstr. (2006) in press.

[8] D.N. Mastronarde, J. Struct. Biol. 120 (1997) 343.

[9] We thank Drs. R. Harmon, S. Mayer and J.A. Hunt of Gatan for giving valuable advice, and also for providing a beta version of the Gatan EFTEM tomography software running with Digital Micrograph and GMS.

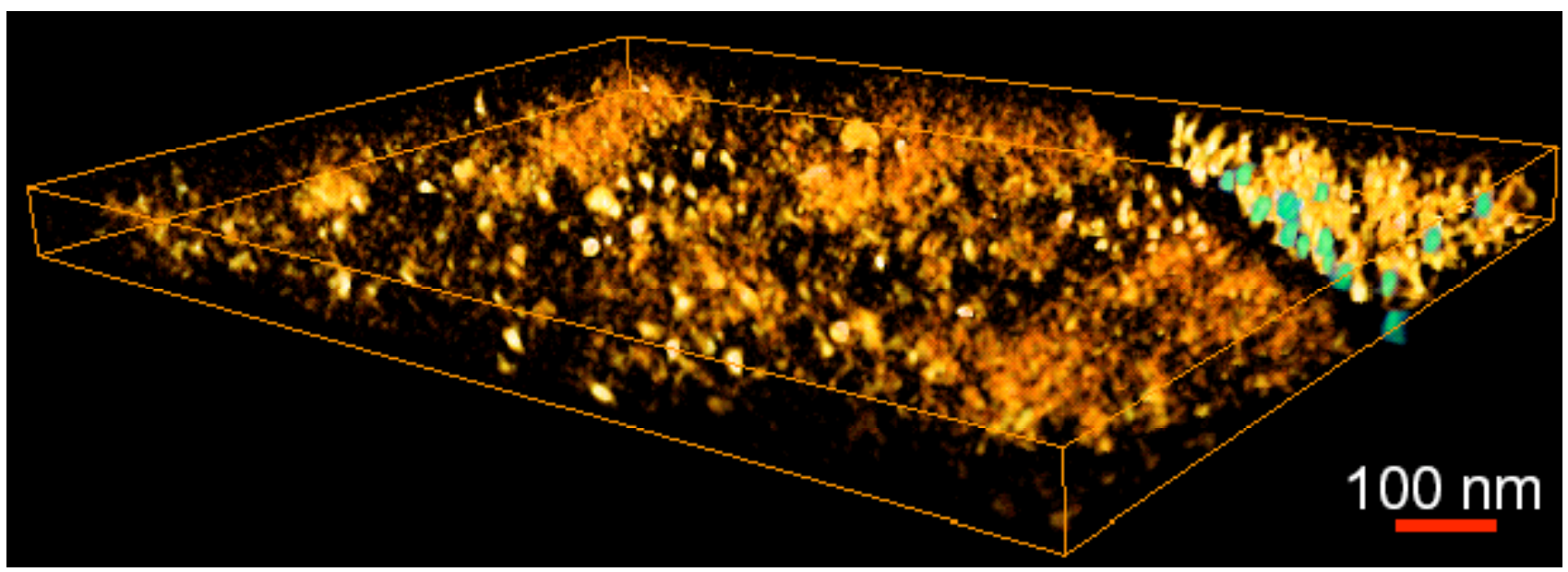

Fig. 1. Three-dimensional phosphorus distribution in unstained plastic section of high-pressure frozen and freeze-substituted Drosophila larval cell, showing part of nucleus and a region of cytoplasm containing ribosomes (top right); the phosphorus distribution reflects the distribution of nucleic acid. 